\title{
Metabolic and electrophysiological validation of functional MRI
}

T Krings, M Schreckenberger, V Rohde, H Foltys, U Spetzger, O Sabri, M H T Reinges, S Kemeny, P T Meyer, W Möller-Hartmann, M Korinth, J M Gilsbach, U Buell, A Thron

\begin{abstract}
Objectives-Although functional MRI is widely used for preoperative planning and intraoperative neuronavigation, its accuracy to depict the site of neuronal activity is not exactly known. Experience with methods that may validate fMRI data and the results obtained when coregistering fMRI with different preoperative and intraoperative mapping modalities including metabolically based ${ }^{18} \mathrm{~F}$-fluorodeoxyglucose PET, electrophysiologcally based transcranial magnetic stimulation (TMS), and direct electrical cortical stimulation (DECS) are described.

Methods-Fifty patients were included. PET was performed in 30, TMS in 10, and DECS in 41 patients. After coregistration using a frameless stereotactic system, results were grouped into overlapping $(<1$ cm distance), neighbouring $(<2 \mathrm{~cm})$, or contradictory $(>2 \mathrm{~cm})$.
\end{abstract}

Department of Neuroradiology, University Hospital of the Technical University,

Pauwelsstrasse 30, 52057 Aachen, Germany

T Krings

S Kemeny

W Möller-Hartmann

A Thron

Department of

Nuclear Medicine

M Schreckenberger

O Sabri

P T Meyer

U Buell

Department of

Neurosurgery

T Krings

V Rohde

U Spetzger

M H T Reinges

$M$ Korinth

J M Gilsbach

Department of Neurology and

Interdisciplinary

Center for Clinical

Research-Central

Nervous System

H Foltys

Correspondence to: Dr T Krings

tkrings@izkf.rwth-aachen.de

Received 31 May 2001 Accepted 27 July 2001
Results-Comparing fMRI with PET, 18 overlapping, seven neighbouring, and one contradictory result were obtained. In (because of motion artefacts, low signal to noise ratio, and unusual high tumour metabolism in PET). The comparison of TMS and fMRI showed seven overlapping and three neighbouring results. In three patients no DECS results could be obtained. Of the remaining 38 patients, fMRI hand motor tasks were compared with DECS results of the upper limb muscles in 36 patients, and fMRI foot motor tasks were compared with DECS results of the lower limb on 13 occasions. Of those 49 studies, overlapping results were obtained in 31 patients, and neighbouring in 14. On four occasions fMRI did not show functional information (because of motion artefacts and low signal to noise).

Conclusions-All validation techniques have intrinsic limitations that restrict their spatial resolution. However, of 50 investigated patients, there was only one in whom results contradictory to fMRI were obtained. Although it is not thought that fMRI can replace the intraoperatively updated functional information (DECS), it is concluded that fMRI is an important adjunct in the preoperative assessment of patients with tumours in the vicinity of the central region.

(f Neurol Neurosurg Psychiatry 2001;71:762-771)

Keywords: functional MRI; presurgical planning; motor cortex four patients no comparison was possible
Although most of the problems of functional magnetic resonance imaging (fMRI) such as low signal to noise ratio, ${ }^{1}$ motion related artefacts, ${ }^{2}$ and signal from large draining veins ${ }^{3}$ can be sufficiently detected and in some cases even reduced or eliminated, ${ }^{4}$ the accuracy of fMRI is still not exactly known. Because fMRI is widely used for presurgical planning of tumours in the vicinity of cortical motor regions, ${ }^{5-12}$ validation of this technique should therefore be performed to quantify its localisation accuracy. One means to assess the validity of fMRI is to combine fMR data with the results of other imaging modalities - that is, positron emission tomography (PET), ${ }^{13}$ transcranial magnetic stimulation (TMS), ${ }^{14}$ or direct electrical cortical stimulation (DECS). ${ }^{16}{ }^{17}$ The goal of this study was (a) to describe the methods that can be used to coregister these different brain mapping modalities and, therefore, to be able to validate fMR and $(b)$ to estimate the localisation accuracy of fMRI by comparing its results with the other, above mentioned methods in a large series of neurosurgical patients.

\section{Methods}

Between May 1997 and February 2000 we investigated, using fMRI, 103 patients with a mass lesion near the central region distorting normal cortical anatomy by displacing the central sulcus. ${ }^{4}$ In some of these patients, additional brain mapping modalities, either preoperatively or intraoperatively, were employed. This paper describes the following subsets of patients: (a) patients in whom fMRI and PET were performed $(\mathrm{n}=30),(b)$ patients who underwent fMRI and transcranial magnetic stimulation $(n=10)$, and (c) patients whose intraoperative DECS mapping was documented in addition to their preoperative fMRI examinations $(n=41)$. Because some patients underwent more than one mapping procedure, a total of 50 patients were included in this study. Patient age ranged from 21 to 77 years (mean 50.2 years), 22 patients were men, tumour histology included 13 patients with glioblastoma multiforme, 10 patients with meningioma, nine with solitary cerebral metastases, four patients with astrocytoma World Health Organisation (WHO) $\mathrm{III}^{\circ}$, three patients with a hamartoma, two patients each with oligoastrocytoma $\mathrm{III}^{\circ}$ and $\mathrm{II}^{\circ}$, anaplastic meningeoma, and one patient each with oligodendroglioma grades II and III, oligoastrocytoma grade II, cavernoma, and a calcified aneurysm. 
fMRI

All imaging studies were performed on a $1.5 \mathrm{~T}$ Philips Gyroscan NT (Philips Medical Systems, Best, Netherlands) using a standard headcoil. After localising images in three planes, strongly $\mathrm{T} 1$ weighted multishot inversion recovery EPI images were obtained for anatomical reference (Imaging parameters: TR/TE/TI: 2000/22/400, FOV: $220 \times 220$, matrix: $256 \times 256$, slice thickness $7 \mathrm{~mm}$ ). Images covered the whole brain and were oriented parallel to the line running through the anterior and the posterior commissure. Additionally, a high resolution $3 \mathrm{D}$ FFE (fast field echo) $\mathrm{T} 1$ weighted anatomical dataset was obtained for subsequent coregistration with other imaging modalities (discussed later, fig 1) (imaging parameters: TR/TE/FA 30/4.6/30, FOV $220 \times 220$, matrix $256 \times 256$, slice thickness 2 $\mathrm{mm})$. Functional images were obtained with the same slice number, orientation, and localisation as the IR-EPI images using a blood oxygenation level dependent (BOLD) single shot multislice $\mathrm{T} 2^{\star}$ weighted gradient echo EPI sequence (TR/TE/FA 4000/40/40, FOV $220 \times 220$, matrix $64 \times 64$, slice thickness $7 \mathrm{~mm}$ ). In a total scanning time of 4 minutes 24 seconds six alternating epochs of rest and activation each lasting 44 seconds were performed by all patients. The scan comprised a total volume of 66 time points with a temporal resolution of 4 seconds/time point. We used boxcar paradigms contrasting motor activation with rest. The motor paradigm was chosen with respect to tumour localisation and motor deficits. The following tasks were performed by the patients: (a) finger opposition using the affected hand to carry out a self paced light touch of thumb pad to finger pad of each subsequent finger without looking at the hand (finger tapping), (b) repetitive self paced closing of the hand to a fist (hand clenching), (c) repetitive flexion of the toes (toe curling), (d) alternating foot flexion and extension (foot flexion). In this series there were no patients present in whom we tested the facial muscles. Sites of functional activation were statistically evaluated on a voxel by voxel basis using the Kolmogorov-Smirnov non-parametric statistical test to compare the MR signal time course with the given task paradigm. Transition times (two time points $=8$ seconds after a signal was given) were not included in the statistical evaluation to account for the delayed haemodynamic response curve. The software calculated the activation maps, which were subsequently colour coded on a thermal scale according to the level of statistical significance concerning the difference between rest and activation states and overlaid over the anatomical inversion recovery EPI images. Whereas the low threshold for activation was set at $\mathrm{p}<0.005$ in all cases, the high threshold varied interindividually according to the varying percentage signal change between patients. If motion artefacts were present in a functional study, a
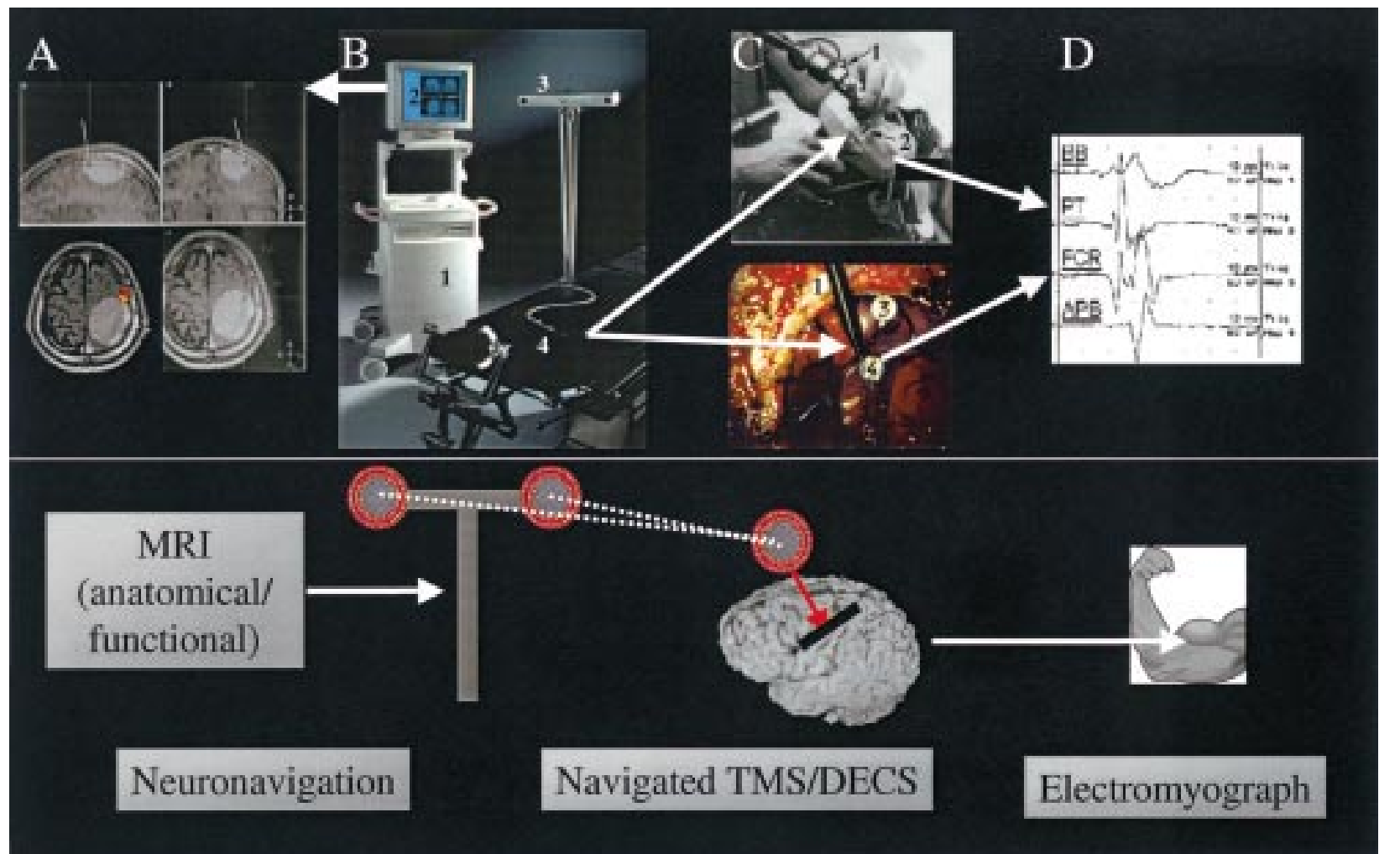

Figure 1 Coregistration of functional MRI and electrophysiological techniques. (A) Functional and anatomical MRI are present in a neuronavigation environment. The neuronavigation system (B) consists of a computer workstation (1) where the MRI of the individual patient is displayed in three dimensions (2). This workstation is connected to an infrared based optical transmitter system (3) that continuously identifies the position of the pointer (4) in space. After coregistering the patient's head to the MRI employing fiducials pasted on the head, the position of the pointer can be followed in real time on the neuronavigation system's display. The pointer (1) is connected to the transcranial magnetic stimulation coil (2) (C, upper image) or identifies the cortical region where maximal compound muscle action potentials (D) are registerd by EMG. A direct comparison of different brain mapping modalities is thus possible. 
motion detection and correction algorithm was used to realign images. Scans with severe motion artefacts $(>2 \mathrm{~mm}$ shift in head position) were excluded. The distance between the peak/maximum of the described complimentary imaging modality and the fMRI peak was rated as overlapping ( $<1 \mathrm{~cm}$ distance), neighbouring ( $<2 \mathrm{~cm}$ distance) or contradictory ( $>2 \mathrm{~cm}$ distance).

PET

Examinations by PET (rest and activation) were performed on an ECAT exact tomograph (Siemens/CTI, Erlangen/Knoxville, Germany/ USA). All patients fasted for a minimum of 10 hours before the study. After injection of 122 $301 \mathrm{MBq}{ }^{18} \mathrm{~F}$-fluorodeoxyglucose $\left({ }^{18} \mathrm{FDG}\right)$, patients were instructed to lie in the supine position in a darkened room with eyes closed and with minimal background noises. Thirty minutes after the injection, the patient was placed in the PET scanner and the head fixed with an elastic latex strip and an emission scan was acquired for 15 minutes. Attenuation correction was performed using a computerised threshold limit routine to define an isodensity contour for the maximum cerebral activity/ pixel. The exact position of these isodensity contours were controlled visually slice by slice and eventually corrected manually. Next, 47 transversal slices (slice thickness $3.375 \mathrm{~mm}$ ) were reconstructed using a Hanning filter (cut off frequency 0.5 ). Transaxial full width at half maximum (FWHM) was $6.0 \mathrm{~mm}$.

The second PET was performed (either some hours later on the same day or on the next day) under a specific motor activation task similar to the paradigm performed during fMRI. The activation tasks were started simultaneously with a second ${ }^{18}$ FDG injection and lasted 30 minutes. Subsequently, an emission scan was acquired for 15 minutes after the motor activation was finished. Attenuation correction and image reconstruction was performed as described for the resting scan (see above).

After AC/PC (anterior/posterior commissura) alignment of the three dimensional MRI data set, the PET data sets (resting scan and activation scan) were realigned to fit the MRI data using a special multimodal image processing software (MPI Tool) ${ }^{18}$ under isodensity contour definition in the PET data sets. Next, the two PET examinations were normalised to each other as a proportional scaling after the global cerebral activities at rest and under activation were calculated using an automatic, threshold value based segmentation.

Next, parametric division (activation divided by rest) and subtraction images (activation minus rest) were calculated and the resultant activated areas projected into the MRI data set (PET/MRI overlay) according to empirical threshold values. Brain voxels were considered as significantly activated if the ratio regional normalised counts of the activation study divided by the regional normalised counts of the resting study was higher than 1.1 . This categorical threshold value of $10 \%$ was set according to increases in blood flow and glucose metabolism, which are characteristic for motor tasks, as evident from data of our own and from the literature.

\section{NEURONAVIGATION}

Both electrophysiological brain mapping modalities (TMS and DECS) were coregistered to the MRI data via a frameless stereotactic navigation system (Easy Guide Neuro, Philips Medical System, Best, The Netherlands). This system consists of a mobile workstation, an optical localising system with two infrared sensitive cameras, and a pointing device with three infrared light emitting diodes. The preprocedurally obtained anatomical three dimensional MRI data set of the patient was loaded on the workstation while the patient's head was fixated. The patient's head was spatially coregistered to the MRI by means of external fiducial markers which were identified both on the MRI dataset after imaging was completed and on the head during the TMS session or the operation. By touching the fiducials on the head of the patient with the pointing device, the spatial coordinates (as measured by the infrared diodes) and the image coordinates (as given from the MRI) were matched. ${ }^{19}$ From those two sets of coordinates a matrix transformation was calculated which allowed the computer workstation to display the coordinates of the arm tip on the three dimensional MRI in real time.

TMS

Compound muscle action potentials (CMAPs) were recorded with surface electrodes over muscles that were primarily involved in the functional MRI tasks (abductor pollicis brevis (APB) for the finger opposition task). Transcranial magnetic stimulation was performed using a Magstim 200 magnetic stimulator (Magstim Company Ltd, UK) with a flat figure 8 coil at $10 \%$ over resting motor threshold of the investigated muscle. The coil was placed on the head so as to cause an induced electrical current flow from posterior to anterior perpendicular to the central sulcus. ${ }^{20}$ The patients' heads were immobilised while they were seated in a reclining chair. Recordings were performed simultaneously for $100 \mathrm{~ms}$ after the stimulus with the muscle at rest, filtered $(5 \mathrm{kHz}-20 \mathrm{~Hz})$, amplified (gain of $500 \mu \mathrm{V} /$ division), digitised, and stored for off line analysis. Peak to peak amplitudes of six stimuli applied to a single stimulation site were averaged. The stimulating coil was rigidly attached to the neuronavigation system. As the TMS coil was moved over the patient's head, the computer workstation of the neuronavigation system graphically displayed the position of the pointer tip in axial, coronal, and sagittal reconstructions of the patient's MRI in real time. The perceived length of the distal pointer tip was modified in the neuronavigation system's software so that when the coil was applied to the scalp, the computer displayed the location on the cortical surface where the peak electric field and therefore stimulation was most likely to occur. ${ }^{21}$ The cortical areas defined as the peak TMS response were obtained by moving the coil in 
steps of $0.5 \mathrm{~cm}$ over the presumed motor areas while stimulating six times over each stimulation site. Real time visualisation of the stimulated area allowed us to stimulate exactly the same area during the train of six stimuli. By comparing the thereby obtained CMAP amplitude with the previous stimulation site amplitude the operator was able to perceive whether the new stimulation site was closer or further away from the epicentre of the investigated muscle. Using this technique, we were usually able to identify the peak stimulation site after testing 15-20 cortical sites.

DECS

Direct electrical cortical stimulation data were also coregistered to the functional MRI data via the frameless stereotactic navigation system that was routinely employed in the operating room for neuronavigation. After coregistration of this system to the patient's head the computer workstation displayed the position of the pointer on three orthogonal slices on the patient's MRI. Intraoperative data were only used if the localisation accuracy of the system was better than $5 \mathrm{~mm}$. Data with an accuracy lower than $5 \mathrm{~mm}$ were not further evaluated. All patients were operated on under general anaesthesia without muscle relaxants. Intraoperatively, electrophysiological monitoring of the unconscious patient was performed after incision of the dura with a monopolar anodal electrical stimulus, delivered from a constant voltage stimulator through a silver plate electrode of $1 \mathrm{~cm}$ diameter. One train of stimuli contained 5 pulses each of $100 \mu$ s duration. ${ }^{22}$ Compound muscle action potentials were recorded simultaneously from eight muscles of the lower and upper limbs via subcutaneous needle electrodes (Viking IV electromyograph, Nicolet Biomedical Instruments, Madison, WI, USA, Filter settings were between 30 and $3000 \mathrm{~Hz}$, sensitivity was set between 10-50 $\mu \mathrm{V} /$ division). Routinely, we selected four muscles of the upper limb (abductor pollicis brevis, flexor carpi radialis, biceps brachii, triceps brachii), and four muscles of the lower limb (quadriceps femoris, adductor longus, tibials anterior, abductor hallucis). Stimulus intensity was increased stepwise until compound muscle action potentials were elicited or the limit of 25 $\mathrm{mA}$ was reached. ${ }^{23}$ Cortical areas from which the largest CMAPs were evoked, were identified using the frameless stereotactic system and marked within the three dimensional MRI data set. Thus, a direct comparison between direct electrical cortical stimulation and fMRI response pattern was possible. The distance between the parenchymal fMRI activation and the cortical area identified during direct electrical cortical stimulation was measured and categorised.

\section{Results}

All procedures were well tolerated by all patients. No undesired side effects were found, either during or after functional imaging or stimulation.
fMRI

The 50 patients comprising this study performed 70 functional runs during fMRI scanning. Twenty seven patients performed hand clenching tasks (mean percentage signal change $2.56 \%$, SD $0.74 \%$, range $1.7-4.2 \%$, two failures due to motion artefacts, one failure due to low signal to noise ratio). In five patients the results of the hand clenching tasks were compared with TMS results, in 12 patients compared with PET, and in 20 patients with DECS (in two of these, no DECS responses could be obtained; see below).

Twenty three patients performed finger opposition tasks (mean percentage signal change $2.72 \%$, SD $0.90 \%$, range $1.9 \%-4.3 \%$, one failure due to motion artefacts, one failure due to low signal to noise ratio). In five patients the results of the finger opposition tasks were compared with TMS results, in 12 patients compared with PET and in 19 patients with DECS (in one of these, no DECS responses could be obtained; see below).

Eight patients performed foot flexion and extension tasks (mean percentage signal change $2.30 \%$, SD $0.63 \%$, range $1.6 \%-3.4 \%$ ). In six patients each the results were compared with PET and DECS.

Twelve patients performed toe curling tasks (mean percentage signal change $2.24 \%$, SD $0.43 \%$, range $1.8 \%-2.9 \%$, one failure due to motion artefacts). In eight patients the results of the toe curling tasks were compared with DECS (in one of these, no DECS responses could be obtained; see below).

PET

In 30 patients the results of fMRI were compared with those obtained by FDG-PET. Four patients had to be excluded from data comparisons because in two of them, severe motion artefacts were present on fMRI, in one patient PET did not show any significant parenchymal activation due to excessive tumour metabolism and in one patient neither fMRI nor PET was able to demonstrate cortical activation due to a low signal to noise ratio. Of the remaining 26 patients, 18 patients had overlapping results of fMRI and PET (same gyrus, $<1 \mathrm{~cm}$ distance, fig 2 ), seven patients had neighbouring activation peaks (gyrus and adjacent sulcus, between 1 and $2 \mathrm{~cm}$ distance) and one patient had contradictory fMRI and PET results (activation on different gyri or $>2$ $\mathrm{cm}$ apart from each other). This patient demonstrated activation in the hemisphere ipsilateral to the hand movement during PET and contralateral activation (ipsilateral to the lesion hemisphere) during fMRI. In four of the seven patients in whom neighbouring results were obtained, fMRI activation projected upon the central sulcal vein whereas PET demonstrated parenchymal activation within the precentral gyrus (fig 3), in two patients moderate motion artefacts were present that projected on the precentral sulcus $(n=1)$ and the central sulcus $(n=1)$ with no correspondence to the central sulcal vein and in one patient activation maximum during PET was within the tumour but not within the parenchyma as 

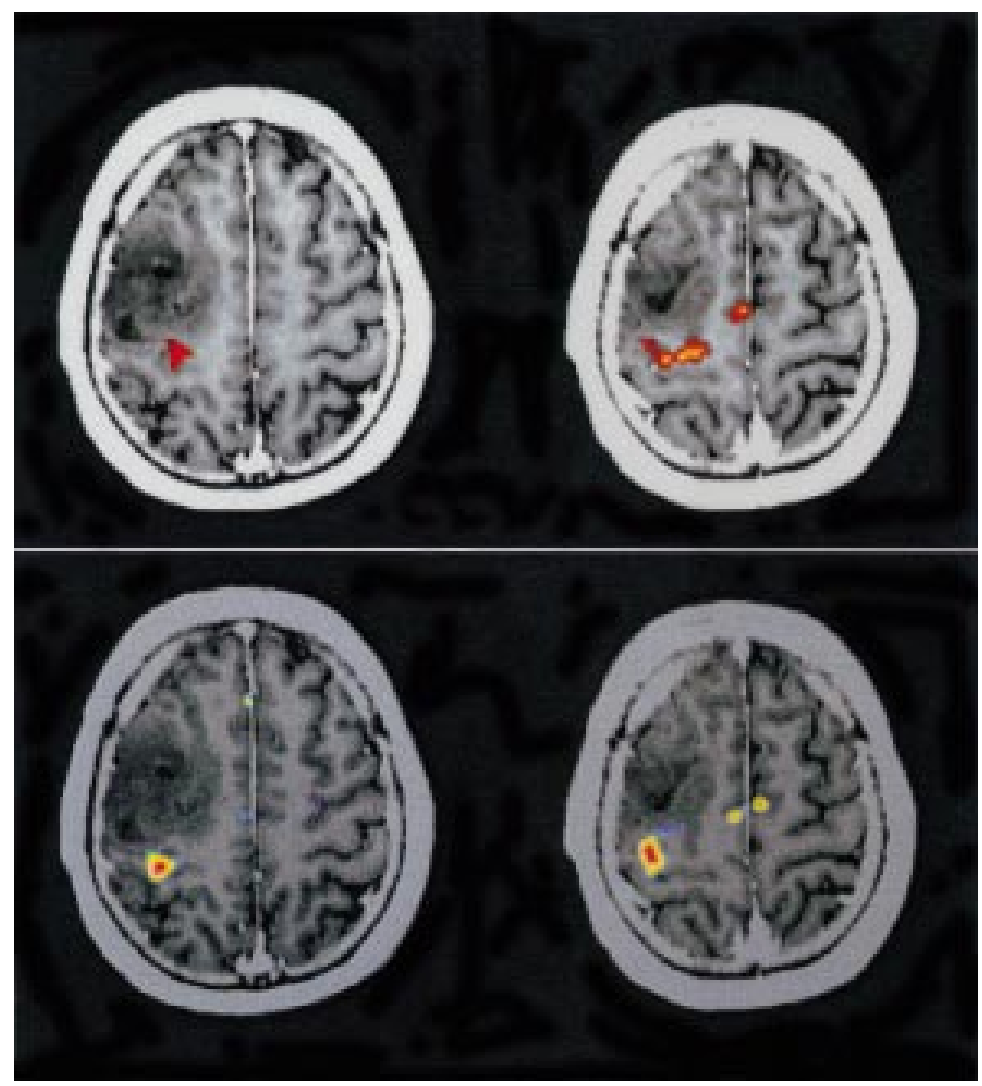

Figure 2 Comparison of $F M R I$ and PET during/after finger tapping of the left hand. (upper row: fMRI, lower row: PET). In this patient with a mild paresis of his left upper limb, activation is seen in the primary motor cortex and the supplementary motor area in both modalities in exactly matching cortical regions. was shown by fMRI. Of the 26 patients, muscles of the lower limb were tested in six (foot flexion and toe curling), here we found three overlapping and three neighbouring results, muscles of the hand were investigated in 24 patients, here we found 15 overlapping, four neighbouring, and one patient with contradictory results, in four patients no comparisons were possible (already discussed).

NEURONAVIGATION

The error induced by the neuronavigation system that was both used for TMS and for depiction of the DECS results on the MR dataset was computed by the system automatically after registration as the discrepancy between the actual spatial localisation of the landmarks on the patient compared with that predicted by MRI in all three dimensions. For the ten TMS measurements the mean error was $0.25 \mathrm{~cm}$ (SD $0.07 \mathrm{~cm}$, maximum error: $0.33 \mathrm{~cm}$ ). During the DECS measurements in open brain surgery, error induced by the FSS had similar values with a mean error of 0.33 $\mathrm{cm}$. In no case was the error larger than $0.4 \mathrm{~cm}$.

TMS

Compound muscle action potentials (CMAPs) after TMS could be obtained in all 10 patients for the investigated hand muscle (abductor pollicis brevis). Cortical sites of stimulation were identified with the neuronavigation system and displayed on the patients' MR data. We found a gradual decay in amplitude when stimulating at sites progressively distant from the peak TMS activation site. Surrounding the peak amplitude there was usually a larger area
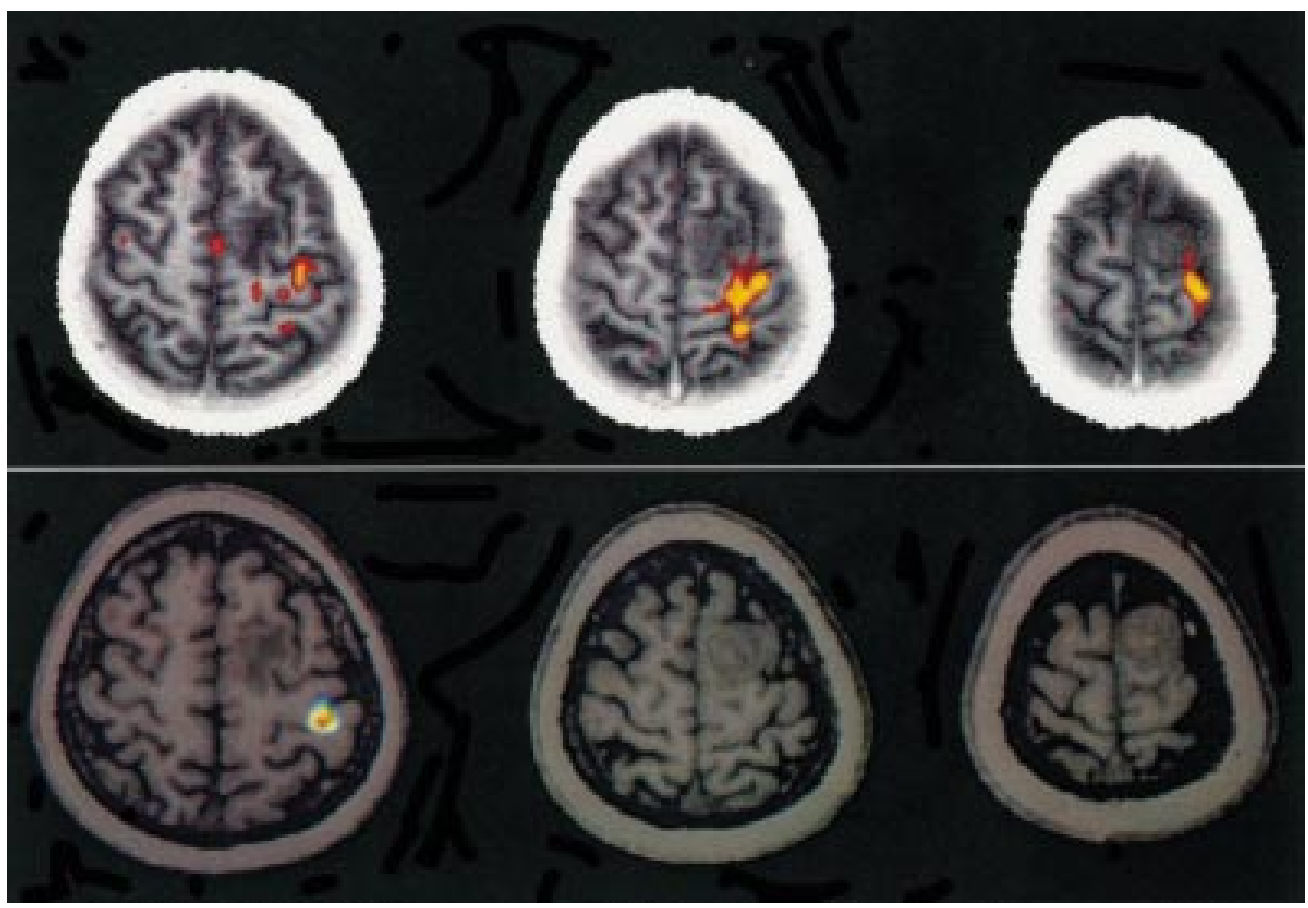

Figure 3 Comparison of $f M R I$ and PET during and after finger tapping of the left hand (upper row fMRI, lower row $P E T)$. In this patient one of the shortcomings of the haemodynamically based fMRI compared with the metabolically based FDG PET is visualised: the demonstration of large draining veins in addition to the demonstration of small parenchymal venules within the primary motor cortex. Although the parenchymal activation of fMRI (left image) matches the PET activation within the same slice, additional "activation" is seen during fMRI in adjacent superior slices, most likely resembling a large draining vein within the central sulcus. No metabolic activation can be seen during PET within these areas. 

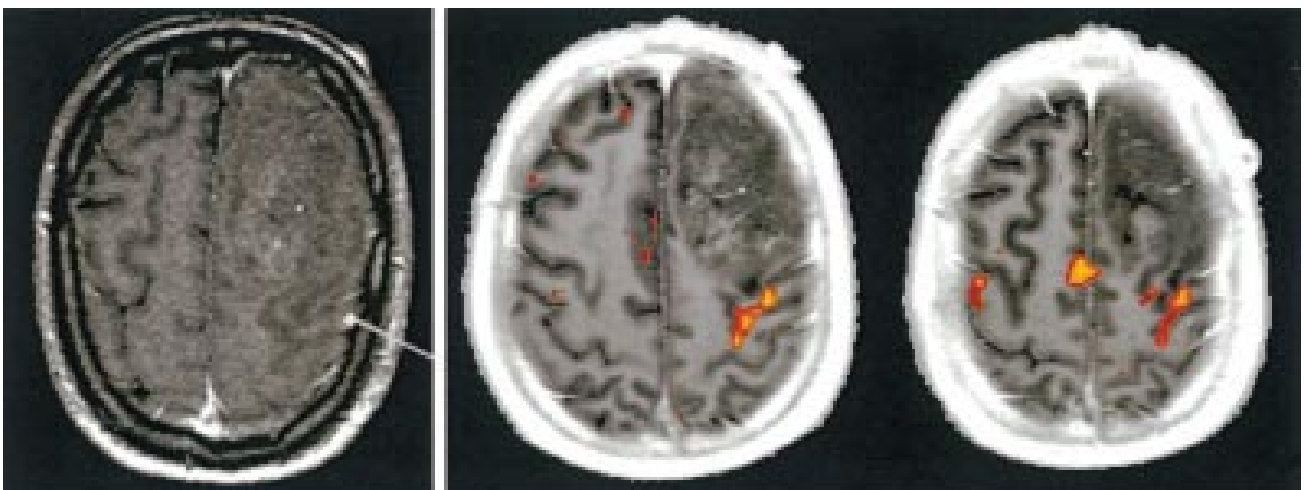

Figure 4 Comparison of fMRI (finger tapping) and TMS. The image on the left demonstrates the cortical area identified by the neuronavigation system from which the maximum compound muscle action potentials (CMAPS) of the abductor pollicis brevis muscles were elicited during TMS. These results compare favourably with the fMRI results during finger tapping (during which the muscle investigated during TMS is predominantly active).

of low amplitude responses. The distances between the peak parenchymal fMRI activation after motor hand activation and the cortical area where TMS elicited the maximum CMAPs of the investigated small hand muscle ranged between 0 and $1.2 \mathrm{~cm}$ (mean $0.6 \mathrm{~cm}$ $(\mathrm{SD} 0.4) \mathrm{cm})(\mathrm{fig} 4)$. Contradictory results $(>2$ $\mathrm{cm}$ difference between both techniques) were not obtained in any patient, seven patients had overlapping results, three had neighbouring stimulation/activation peaks. In these three, peak fMRI activation projected on more medial (deep) cortical areas, whereas TMS sites projected on the cortical surface.
DIRECT ELECTRICAL CORTICAL STIMULATION (DECS)

In 41 patients DECS was documented, in three patients no CMAPs could be elicited with a stimulus intensity of $25 \mathrm{~mA}$ maximum. Of the remaining 38 patients, hand motor tasks during fMRI were compared with DECS results of the upper limb muscles in 36 patients (fig 5); foot motor tasks were compared with DECS results of the lower limb on 13 occasions; in 11 patients both upper and lower limb muscles were tested during fMRI and DECS. In two patients, severe motion artefacts

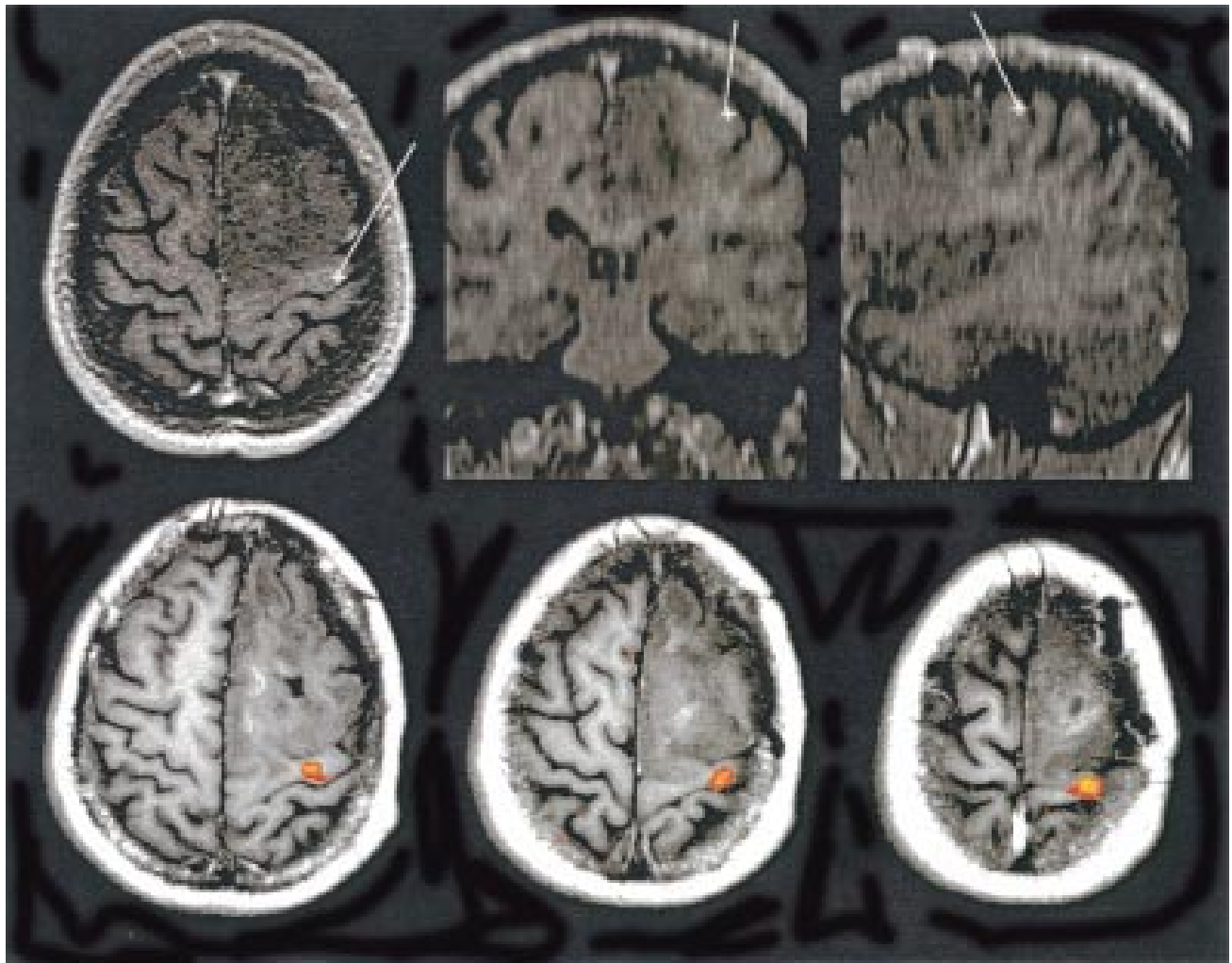

Figure 5 Comparison of fMRI (finger tapping) and direct electrical cortical stimulation (DECS). The upper row demonstrates the position of the pointer that identified the cortical region from which the maximum compound muscle action potentials (CMAPs) of the small hand muscles were elicited during DECS. Although the stimulated area closely matches the parenchymal activation visible on the left slice, additional "activation" is visible within the central sulcus representing a large draining vein that should not be mistaken for active cortical neurons. 
were present during the finger and hand movement tasks that rendered comparison with DECS impossible; in one patient, motion artefacts in the lower limb fMRI tasks made comparisons with DECS impossible; in one patient a low signal to noise ratio prevented comparison of the upper limb muscles. Thus, 33 upper limb and 12 lower limb locations were compared. Contradictory results $(>2 \mathrm{~cm}$ difference between both techniques) were not obtained in any patients, 31 patients (26 tested upper limbs and five tested lower limbs) had overlapping results, on 14 occasions, neighbouring stimulation/activation peaks were found (seven tested upper and lower limbs each).

\section{Discussion}

METABOLIC VALIDATION USING ${ }^{18}$ FDG-PET

Previous studies on PET based preoperative brain mapping studies relied on freely diffusing radiotracers such as ${ }^{15} \mathrm{O}$ water or ${ }^{15} \mathrm{O}$ butanol. ${ }^{25-28}$ Major advantages of these radiotracers are the possibility of performing multimodal activations and the better temporal resolution. However, ${ }^{18}$ FDG PET has the advantage of a higher spatial resolution due to lower positron energy $\left({ }^{18} \mathrm{~F} 0.64 \mathrm{MeV}\right.$, medium range $0.2 \mathrm{~mm} ;{ }^{15} \mathrm{O} 1.72 \mathrm{MeV}$, medium range $1.5 \mathrm{~mm}$ ), a better signal to noise ratio, ${ }^{29}$ and a more wider availability as it does not require an on site cyclotron. Moreover, it detects metabolism instead of blood flow. In addition to brain activation studies this protocol allows for assessing tumour metabolism as a prognostically significant grading parameter. ${ }^{30} 31$ Preoperative PET activation using ${ }^{18} \mathrm{FDG}$ has a high reliability and validity of localising functional cortex. ${ }^{32}{ }^{33}$ We found a good correspondence of the PET findings with the fMRI data in all but one patient. In this patient, fMRI localised the activation peak within the precentral gyrus of the affected hemisphere. Although PET also demonstrated activation close to the area demonstrated by fMRI, its main activation peak was visible on the hemisphere contralateral to the brain tumour. We interpret these findings as a lesion dependent reorganisation with activation both in the primary motor cortex (M1) of the affected hemisphere and additional or "secondary" motor areas, including contralateral M1. After and during recovery of a lost function, plastic changes may occur that lead to shifts of activation to homologous areas of the unaffected hemisphere. ${ }^{34}$ In patients with brain tumours, a contribution of the contralateral intact M1 has been found. ${ }^{35}{ }^{36}$ In our patient, bilateral activation was visible during PET and fMRI with the peak activation, however, on different hemispheres. It might be presumed, therefore, that in this patient lesion induced changes with bilateral activation have taken place and that the secondary motor areas were activated to varying degrees.

Possible reasons for the relatively high incidence of neighbouring instead of overlapping results of fMRI and PET data include (1) head movement artefacts during fMRI that did not occur during the ${ }^{18} \mathrm{FDG}-\mathrm{PET}$ protocol (PET started after the motor activation was finished), and (2) abnormally increased tumour metabolism visible during PET that obscured activation within adjacent grey matter. The most common reason, however, for neighbouring activation in our series was a more prominent activation within the central sulcus during fMRI compared with the parenchymal activation visible in PET. When overlaying our results on contrast enhanced studies, it became obvious that fMRI depicted the central sulcal vein in these cases rather than the active parenchyma of the precentral gyrus. Functional MRI depicts neuronal activity indirectly via haemodynamic changes in the human brain. Vessels draining the site of neuronal activation, not the metabolically active neurons, are revealed. We have found a considerably higher percentage signal change in large draining veins compared with parenchymal venules. ${ }^{37}$ As large draining veins with an unclear relation to the site of neuronal activity have larger percentage signal changes, caution must be taken if a study shows a low signal to noise ratio. Parenchymal activation yields relatively lower percentage signal changes (typically in the order of $2.5 \%$ but in some patients as low as $1.6 \%$ ). ${ }^{1}$ If background noise (typically in the order of $1.2 \%$ but in some studies as high as $1.9 \%$ ) approximates to the parenchymal activation percentage signal change, this parenchymal percentage signal change might be too low to yield statistically significant results. In these cases parenchymal activation with a lower statistical power might even not be detectable whereas venous activation still is. This leads to misinterpretation of the anatomical correlate of the specific task related activity as only larger venous structures demonstrate a high enough percentage signal changes and therefore a high enough statistical power.

NEURONAVIGATION FOR COREGISTERING FMRI WITH ELECTROPHYSIOLOGICAL TECHNIQUES The utility of both electrophysiological methods for validating fMRI depends on the spatial resolution of the electrophysiological procedures and the application error of the method by which the different modalities are integrated-that is, the intrinsic error of the neuronavigation system. The accuracy of the system was always better than $4 \mathrm{~mm}$, at least at the beginning of the procedure. The localisation error of the frameless stereotactic device primarily resulted from inaccuracies in the identification and matching of the fiducials on the MRI and the subject's head. Patient movements during TMS and traction upon the fiducials after fixation of the head within the Mayfield clamp during DECS typically result in larger errors. Multiple recalibrations and retesting of the accuracy can diminish the movement induced inaccuracy. Because the neuronavigation systems are not yet capable of adapting to the permanently changing anatomical situation during the operation, a progressive loss of accuracy during debulking of the lesion is inevitable. ${ }^{19}$ Deformation of brain structures of more than $10 \mathrm{~mm}$ have been reported ${ }^{38}{ }^{39}$ rendering comparison with preoperative fMRI data based on neuronavigation 
systems impossible. We therefore tried to perform DECS before tumour resection; this was, however, not always possible.

ELECTROPHYSIOLOGICAL VALIDATION USING TMS AND DECS

We have demonstrated the concordance between the haemodynamically based fMRI maps and the electrophysiologically based TMS maps of the finger muscles in 10 patients with space occupying lesions in the central region on the one hand; on the other, we were able to coregister DECS maps of the lower limbs in 12 patients and of the upper limbs in 33 patients with fMRI results. With increasing distance from the presumed central sulcus both the CMAP amplitude and the strength of fMRI activation decreased. Transcranial magnetic stimulation resulted in a more widespread map than did fMRI; on the other hand, fMRI resulted in a more widespread map than did DECS. This is presumably because of the relatively large area of the electric field induced by TMS and the relatively confined electrical field induced by DECS. The possibility that more, less, or different motor neurons are activated cannot be excluded, as the tasks performed during fMRI differ from the induced motor responses in that they are voluntary, self paced, and involve more than those muscles investigated during TMS/DECS. However, the cortical loci corresponding to peak CMAP amplitude after TMS/DECS and peak parenchymal fMRI activation coincided closely even in the setting of a distorting lesion.
Transcranial magnetic stimulation and DECS of the motor system activate cortical areas that are connected to spinal motor neurons directly (DECS) or indirectly (TMS) via excitatory corticocortical interneurons. ${ }^{40}$ The amount of excitatory cortical input determines the number of descending volleys to the spinal cord. Here, the excitatory input is temporally and spatially summated and $\alpha$-motor neurons are recruited according to Henneman's size principle. ${ }^{40}{ }^{41}$ The amplitude of the recorded CMAP is determined by the number of activated spinal motor neurons. Thus, there is a nearly linear relation between the number of activated corticospinal motor neurons and the CMAP amplitude of the respective target muscle. ${ }^{42}$ Mapping the human primary sensorimotor cortex with TMS is therefore based on the relation of the CMAP amplitude elicited over a specific stimulation site to the density of cortical motor neurons being stimulated at that site. The CMAP amplitude increases when stimulating over cortical areas with a higher density of motor neurons.

For TMS, the approximate smallest area of stimulation at rest with a commercially available figure of eight coil (lobe diameter $7 \mathrm{~cm}$ ) is an area of $1-2$ square $\mathrm{cm} .{ }^{43}$ At first glance this area seems too large to yield useful mapping information. However, increased resolution can be obtained by moving the coil in small increments over the scalp and measuring and interpolating the amplitude changes in the

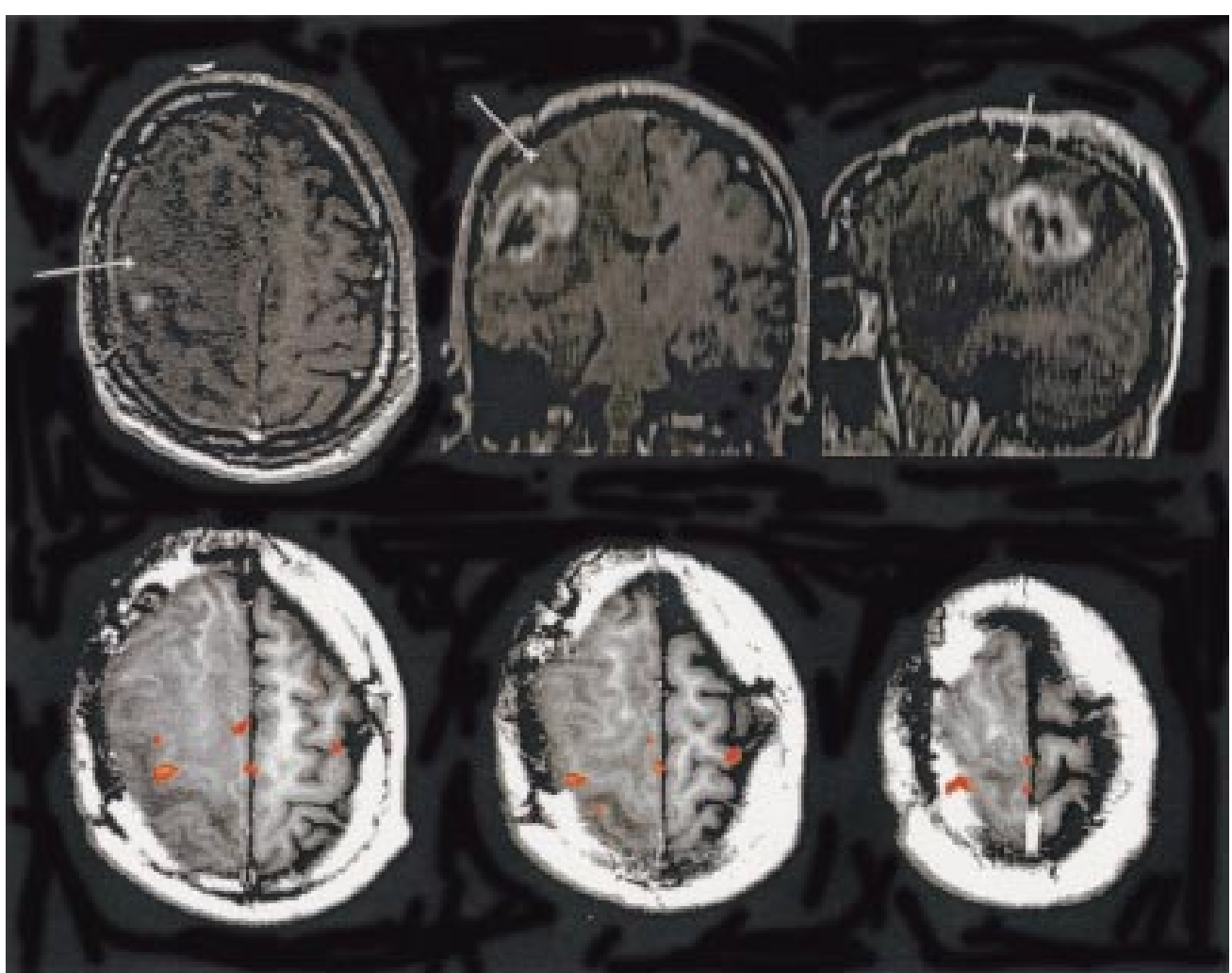

Figure 6 Comparison of $f M R I$ (finger tapping) and direct electrical cortical stimulation (DECS). The upper row demonstrates the position of the pointer that identified the cortical region from which the maximum compound muscle action potentials (CMAPs) of the small hand muscles were elicited during DECS. This image demonstrates one typical shortcoming of DECS - namely, that only the crowns of the gyri can be stimulated whereas the depths of the sulci (in which the main area of activation in this patient was presumed by fMRI) remains inaccessible to the stimulation probe. In addition, this example again demonstrates venous "activation" in superior sections. 
evoked peripheral compound muscle action potential (CMAP). ${ }^{43-46}$ Using our technique, a mapping accuracy of $0.5 \mathrm{~cm}$ can be obtained. ${ }^{21}{ }^{47}$ The spatial resolution of the DECS method we used is within the same scale as we stimulated at points about $0.5 \mathrm{~cm}$ apart from each other. But, although it is possible to stimulate the exposed cortex using DECS, stimulation within the depth of a sulcus is not routinely performed. Therefore, two thirds of the cortical surface cannot be probed using DECS (fig 6). Because of these intrinsic limitations of the localising accuracy of the validation methods themselves we grouped our results into relatively coarse categories (overlapping, neighbouring, and contradictory). It is our opinion that, at present, a higher localisation accuracy cannot be assessed with the standard validation techniques as they do not allow for a finer resolution.

However, there was no systematic error, no systematic shift in position, and, most importantly, we did not find contradictory results between both fMRI/TMS and fMRI/DECS. This allows for validation of fMRI results as follows. It is widely accepted that the regional cerebral blood flow ( $\mathrm{rCBF}$ ) is regulated by the amount of neuronal activity. The magnitude of the change in $\mathrm{rCBF}$ is directly influenced by the size of the activated cortical area and the number of active neurons. ${ }^{158-50}$ Taking these assumptions into consideration cortical areas with a higher density and total number of cortical motor neurons produce a greater increase in regional $\mathrm{CBF}$ and venous oxygenation than cortical areas with a lesser number of motor neurons with a consecutive larger change in $\mathrm{T} 2^{\star} \mathrm{MR}$ signal change that is detected during fMRI. Consecutively, cortical regions with a high statistical power and a larger percentage signal change within the parenchyma should theoretically coincide with the area of largest motor neuron density. This hypothesis was confounded in our study in that the site of greatest motor neuron density, as shown by the peak TMS response and the peak DECS response, was elicited over the area of peak parenchymal fMRI activation in all cases. In a recent study conducted by Hill et $a l^{38}$ the authors critically reviewed the literature on validation of fMRI published so far and reported on their own experience in eight patients. Because of motion related artefacts and brain deformation after craniotomy they found discrepancies in electrophysiologically obtained functional information. Although we agree with these authors that fMRI should be interpreted with caution; that motion artefacts may limit the use of fMRI, and that brain deformation after craniotomy might limit the intraoperative use of fMRI to guide resection, we do not agree with the conclusion that "fMRI was unsatisfactory for localisation of eloquent regions in the vicinity of the sensorimotor cortex". Based on our results of both preoperative (metabolic and electrophysiological) and intraoperative validation techniques we conclude that the parenchymal fMRI response is sufficiently sensitive to demonstrate the active population of motor neurons in the human primary sensorimotor cortex.

\section{Conclusion}

Both metabolically (PET) and electrophysiologically based (TMS, DECS) methods showed concordance with the parenchymal fMRI activation peak, thus validating functional MRI. However, all validation techniques have intrinsic limitations that restrict their spatial resolution. The exact localisation accuracy of functional MRI therefore cannot be given. However, of 50 investigated subjects, there was only one in whom results contradictory to fMRI were obtained. We therefore conclude that fMRI, when interpreted correctly, has a reasonable resolution and is sufficiently accurate for presurgical planning. Therefore this technique is an important adjunct to the preoperative assessment of patients with tumours in the vicinity of the central region. Intraoperatively, however, fMRI data, especially when being linked to a neuronavigation system, should be interpreted cautiously, as brain deformation, EPI related artefacts, and demonstration of flow phenomena during fMRI might lead to substantial-and clinically relevant - shifts in position of functional cortex as was pointed out earlier. ${ }^{38}$ We do not think that fMRI can replace the intraoperatively updated functional information via direct electrical cortical stimulation.

This study was supported by the Deutsche Forschungsgemeinschaft (KR 2008/2-1).

1 Kwong KK. Functional magnetic resonance imaging with echo planar imaging. Magn Reson Q 1995;11:1-20.

2 Hajnal JV, Bydder GM, Young IR. fMRI: does correlation imply activation? NMR Biomed 1995;8:97-100.

3 Frahm J, Merboldt KD, Hanicke W, et al. Brain or vein: oxygenation or flow? On signal physiology in functional MRI
of human brain activation. NMR Biomed 1994;7:45-53.

4 Krings T, Reinges MH, Erberich S, et al. Functional MRI for presurgical planning. Problems, artefacts and solution strategies. F Neurol Neurosurg Psychiatry 2001;70:749-60.

5 Nitschke MF, Melchert UH, Hahn C, et al. Preoperative unctional magnetic resonance imaging (fMRI) of the motor system in patients with tumours in the parietal lobe. Acta Neurochir (Wien) 1998;140:1223-9.

6 Schulder M, Maldjian JA, Liu WC, et al. Functional imageguided surgery of intracranial tumors located in or near the sensorimotor cortex. $\mathcal{F}$ Neurosurg 1998;89:412-8.

7 Pujol J, Conesa G, Deus J, et al. Clinical application of functional magnetic resonance imaging in presurgical identification of the central sulcus. $\mathcal{F}$ Neurosurg 1998;88:863-9.

8 Puce A, Constable RT, Luby ML, et al. Functional magnetic resonance imaging of sensory and motor cortex: comparison with electrophysiological localization. 7 Neurosurg 1995;83:262-70.

9 Lee CC, Ward HA, Sharbrough FW, et al. Assessment of functional MR imaging in neurosurgical planning. AfNR Am 7 Neuroradiol 1999;20:1511-9.

10 Krings T, Reul J, Spetzger U, et al. Functional magnetic resonance mapping of sensory motor cortex for imageguided neurosurgical intervention. Acta Neurochir (Wien) guided neurosurgical

11 Cosgrove GR, Buchbinder BR, Jiang H. Functional magnetic resonance imaging for intracranial navigation. Neurosurg Clin N Am 1996;7:313-22.

12 Achten E, Jackson GD, Cameron JA, et al. Presurgical evaluation of the motor hand area with functional MR maging in patients with tumors and dysplastic lesions. Radiology 1999;210:529-38.

13 Bittar RG, Olivier A, Sadikot AF, et al. Cortical motor and somatosensory representation: effect of cerebral lesions. $\mathcal{F}$ Neurosurg 2000;92:242-8.

14 Boroojerdi B, Foltys H, Krings $\mathrm{T}$, et al. Localization of the motor hand area using transcranial magnetic stimulation and functional magnetic resonance imaging. Clin Neurophysiol 1999;110:699-704.

15 Krings T, Buchbinder BR, Butler WE, et al. Functional magnetic resonance imaging and transcranial magnetic stimulation: complementary approaches in the evaluation of cortical motor function. Neurology 1997;48:1406-16. 
16 Fandino J, Kollias SS, Wieser HG, et al. Intraoperative validation of functional magnetic resonance imaging and cortical reorganization patterns in patients with brain tumors
involving the primary motor cortex. $\mathcal{F}$ Neurosurg 1999;91: involving $238-50$.

17 Lehericy S, Duffau H, Cornu P, et al. Correspondence between functional magnetic resonar totopy and individual brain anatomy of the central region: comparison with intraoperative stimulation in patients with brain tumors. $\mathcal{F}$ Neurosurg 2000;92:589-98.

18 Pietrzyk U, Herholz K, Fink G, et al. An interactive technique for three-dimensional image registration: validation for PET, SPECT, MRI, and CT brain studies. 7 Nucl Med 1994;35:2011-8.

19 Spetzger U, Laborde G, Gilsbach JM. Frameless neuronavi. 1995;38:163-6.

20 Brasil-Neto JP, Cohen LG, Panizza M, et al. Optimal focal transcranial magnetic activation of the human motor cortex: effects of coil orientation, shape of the induced current pulse, and stimulus intensity. F Clin Neurophysiol 1992; rent pulse,

21 Krings T, Buchbinder BR, Butler WE, et al. Stereotactic transcranial magnetic stimulation: correlation with direct electrical cortical stimulation. Neurosurgery 1997;41:131926.

22 Cedzich C, Taniguchi M, Schafer S, et al. Somatosensory evoked potential phase reversal and direct motor cortex stimulation during surgery in and around the centra region. Neurosurgery 1996;38:962-70.

23 Taniguchi M, Cedzich C, Schramm J. Modification of cortical stimulation for motor evoked potentials under genera anesthesia: technical description. Neurosurgery 1993;32: 219-26.

24 Withdrawn

25 Bittar RG, Olivier A, Sadikot AF, et al. Localization of somatosensory function by using positron emission tomography scanning: a comparison with intraoperative cortical raphy scanning: a comparison with intra

26 Duncan JD, Moss SD, Bandy DJ, et al. Use of positron emission tomography for presurgical localization of eloquent brain areas in child

27 Kaplan AM, Bandy DJ, Manwaring KH, et al. Functional brain mapping using positron emission tomography scanning in preoperative neurosurgical planning for pediatric brain tumors. F Neurosurg 1999;91:797-803.

28 Vinas FC, Zamorano L, Mueller RA, et al. $\left[{ }^{15} \mathrm{O}\right]$-water PET and intraoperative brain mapping: a comparison in the

29 Herholz K, Pietrzyk U, Karbe H, et al. Individual metabolic anatomy of repeating words demonstrated by MRI-guided n tomography. Neurosci Lett 1994;182:4750.

30 Meyer PT, Schreckenberger M, Spetzger U, et al. Comparison of visual and ROI based brain tumour grading using 18F-FDG-PET: ROC-analysis. Eur 7 Nucl Med 2001;28: 165-74.

31 Delbeke D, Meyerowitz C, Lapidus RL, et al. Optimal cutoff levels of F-18 fluorodeoxyglucose uptake in the differentiation of low-grade from high-grade brain tumors with PET. Radiology 1995;195:47-52

32 Schreckenberger M, Spetzger U, Sabri O, et al. Localisation of motor areas in brain tumour patients: a comparison of preoperative 18-FDG PET and intraoperative cortical electrostimulation. Eur f Nucl Med 2001;28:1394-1403.

33 Schreckenberger M, Spetzger U, Sabri O, et al. Preoperative PET activation for assessment of motor cortex area in precentral chondroma. Surg Neurol 1999;52:24-9.
34 Nudo RJ, Wise BM, SiFuentes F, et al. Neural substrates for the effects of rehabilitative training on motor recovery after ischemic infarct. Science 1996;272:1791-4.

35 Yoshiura T, Hasuo K, Mihara F, et al. Increased activity of the ipsilateral motor cortex during a hand motor task in patients with brain tumor and paresis. AfNR Am f Neuroradiol 1997; 18:865-9.

36 Alkadhi H, Kollias SS, Crelier GR, et al. Plasticity of the human motor cortex in patients with arteriovenous malformations: a functional MR imaging study. AfNR Am f Neuroradiol 2000;21:1423-33.

37 Krings T, Erberich SG, Roessler F, et al. MR blood oxygenation level-dependent signal differences in parenchymal and large draining vessels: implications for functional MR imaging. A7NR Am 7 Neuroradiol 1999;20: 1907-14.

38 Hill DL, Smith AD, Simmons A, et al. Sources of error in comparing functional magnetic resonance imaging and invasive electrophysiological recordings. $\mathcal{F}$ Neurosurg 2000; 93:214-23.

39 Maurer CR, Hill DL, Martin AJ, et al. Investigation of intraoperative brain deformation using a $1.5-\mathrm{T}$ interventional MR system: preliminary results. IEEE Trans Med Imaging 1998;17:817-25.

40 Day BL, Thompson PD, Dick JP, et al. Different sites of action of electrical and magnetic stimulation of the human brain. Neurosci Lett 1987;75:101-6.

41 Henneman E, Somjen G, Carpenter DO. Excitability and inhibitability of motoneurons of different sizes. 7 Neurophysiol 1965;28:599-620.

42 Kiers L, Clouston P, Chiappa KH, et al. Assessment of cortical motor output: compound muscle action potential versus twitch force recording. Electroencephalogr Clin Neurophysiol 1995;97:131-9.

43 Wassermann EM, McShane LM, Hallett M, et al. Noninvasive mapping of muscle representations in human motor cortex. Electroencephalogr Clin Neurophysiol 1992;85:1-8.

44 Levy WJ, Amassian VE, Schmid UD, et al. Mapping of motor cortex gyral sites non-invasively by transcranial magnetic stimulation in normal subjects and patients. Electroencephalogr Clin Neurophysiol Suppl 1991;43:51-75.

45 Krings T, Naujokat C, von Keyserlingk DG. Representation of cortical motor function as revealed by stereotactic transcranial magnetic stimulation. Electroencephalogr Clin Neurophysiol 1998;109:85-93.

46 Cohen LG, Bandinelli S, Topka HR, et al. Topographic maps of human motor cortex in normal and pathological conditions: mirror movements, amputations and spinal cord injuries. Electroencephalogr Clin Neurophysiol Suppl 1991;43:36-50.

47 Brasil-Neto JP, McShane LM, Fuhr P, et al. Topographic mapping of the human motor cortex with magnetic stimulation: factors affecting accuracy and reproducibility. Electroencephalogr Clin Neurophysiol 1992;85:9-16.

48 Jueptner M, Weiller C. Review: does measurement of regional cerebral blood flow reflect synaptic activity? Implications for PET and fMRI. Neuroimage 1995;2:148-56.

49 Fox PT, Raichle ME. Focal physiological uncoupling of cerebral blood flow and oxidative metabolism during somatosensory stimulation in human subjects. Proc Natl Acad Sci USA 1986;83:1140-4

50 Ibanez V, Deiber MP, Sadato N, et al. Effects of stimulus rate on regional cerebral blood flow after median nerve stimulation. Brain 1995;118:1339-51. 